\title{
High expression of endoplasmic reticulum chaperone grp94 is a novel molecular hallmark of malignant plasma cells in multiple myeloma
}

Saurabh Chhabra ${ }^{1,3}$, Sandeep Jain ${ }^{1,3}$, Caroline Wallace ${ }^{1,2}$, Feng Hong ${ }^{1,2}$ and Bei Liu ${ }^{1,2^{*}}$

\begin{abstract}
Background: Multiple myeloma (MM) is a hematologic malignancy that is characterized by the proliferation of abnormal bone marrow plasma cells (BMPC) and overproduction of immunoglobulin or light chains with evidence of end-organ damage such as bone damage, anemia, hypercalcemia, and renal dysfunction. The pathogenesis of MM is closely linked to dysregulated unfolded protein response (UPR) in the endoplasmic reticulum (ER). Constitutive activation of UPR in mice, as demonstrated by transgenic expression of a master UPR transcription factor XBP1s (a UPR-specific splice variant of X-box binding protein 1), causes myeloma. grp94 (gp96) is a key downstream chaperone in the ER that mediates the UPR as a part of the protein quality control mechanism in the secretory pathway. Our recent study has shown that the persistence of plasma cells as well as the development of myeloma in XBP1s-transgenic mice is critically dependent on grp94. However, the role of grp94 in the initiation and progression of human MM is still unknown.

Methods: The expression level of grp94 in BMPCs was measured by flow cytometry, real-time RT-PCR, and Western blot analysis. We compared the expression levels of grp94 in BMPCs in a spectrum of patients including MM, monoclonal gammopathy of undetermined significance (MGUS), smoldering MM (SMM), as well as non-plasma cell disorders (NPC).
\end{abstract}

Results: We found that grp94 was highly expressed in malignant plasma cells in patients with MM, but not in BMPCs in patients with MGUS/SMM and NPC. The expression level of grp94 correlated significantly with CD138 expression level. We also found that the grp94 expression level in BMPCs from International Staging System (ISS) stage III MM patients is higher than those in ISS stage I/II MM patients.

Conclusions: grp94 is highly expressed in BMPCs in MM, which correlates with the advanced stage of this disease. Our data demonstrated that grp94 is a novel diagnostic and prognostic biomarker. It also positioned grp94 as a promising therapeutic target for MM.

Keywords: Multiple myeloma, Plasma cell, Endoplasmic reticulum, Chaperone, grp94, gp96, Biomarker

\footnotetext{
* Correspondence: liube@musc.edu

${ }^{1}$ Hollings Cancer Center, 86 Jonathan Lucas Street, Charleston, SC 29425, USA

2 Department of Microbiology and Immunology, Medical University of South

Carolina, 86 Jonathan Lucas Street, Charleston, SC 29425, USA

Full list of author information is available at the end of the article
} 


\section{Introduction}

Despite the introduction of new treatments, multiple myeloma (MM) remains an incurable malignant plasma cell disorder [1-11]. It is the second most common hematologic malignancy, and it accounts for $10 \%$ of all hematological malignancies [12-15]. Each year, over 20,000 new cases are diagnosed in the United States of America [16]. MM is a cytogenetically heterogeneous clonal disorder $[13,17,18]$. It typically evolves from an asymptomatic pre-malignant stage called monoclonal gammopathy of undetermined significance (MGUS) [19-21], to an intermediate asymptomatic smoldering MM (SMM) [22], to eventually symptomatic MM. SMM is a biologically heterogeneous entity [23] which includes patients similar to those with MGUS with a very low rate of progression, as well as those who develop clinically evident end-organ damage within the first 2 years of diagnosis [24, 25]. No single pathological or molecular feature can be used to distinguish MM patients from SMM, who have clonal pre-malignant plasma cells from those with clonal myeloma cells.

Durie and Salmon introduced a staging system in 1975 that used M protein, hemoglobin, calcium, and the number of bone lesions to predict MM cell tumor burden [26]. In the 1980 s, serum $\beta 2$-microglobulin $(\beta 2 \mathrm{M})$ was found to be a simple but reliable prognostic marker for staging of MM [27, 28]. Subsequently, albumin [29] and bone marrow plasma cell (BMPC) proliferation indices $[30,31]$ were found to be useful prognostic factors. The International Staging System (ISS) was devised in 2005 using $\beta 2 \mathrm{M}$ and serum albumin level, which enabled staging the patients clinically and ascertaining their prognoses [32]. This has been further refined by combining FISH data with ISS [33, 34]. Other types of biomarkers (including serum free light chain (sFLC) ratio and cytogenetic markers) also provide prognostic information on myeloma and for patients with asymptomatic plasma cell disorders [34-37]. Recent studies showed that CD44 expression is significantly higher in plasma cells from persistent/relapsed MM than untreated patients [38]. Ncadherin protein and gene expression are increased in $\mathrm{CD} 38^{\text {high }} \mathrm{CD} 138^{+}$plasma cells from MM patients, and high expression of $\mathrm{N}$-cadherin correlates with shorter progression-free survival and overall survival comparing with patients with normal N-cadherin level [39]. More recently, it was found that serum miRNA-483-5P is significantly elevated in MM patients, and high expression of miRNA-483-5P is associated with shorter progression-free survival [40]. Insulin-like growth factor binding protein 7 (IGFBP7) expression is associated with the poor prognosis with the absence of myeloma bone disease [41].

The clinical outcome of MM has significantly improved in the last decade due to introduction of the new class of therapeutic agents [42, 43]. Until the 1990s, few advances in treatment of the MM occurred and the median survival of newly diagnosed myeloma patients was 2.5 years [42]. However, beginning in the mid-1990s, with the introduction of high-dose melphalan and autologous hematopoietic cell transplantation (AHCT), survival began to improve [44]. From late 1990s to mid2000s, the overall median survival increased to nearly 4 years [42]. Further improvement in disease control and survival were made in the mid-2000s with the introduction of highly active agents with mechanisms of action independent of DNA damage [42]. Immunomodulatory drugs (IMiDs) such as thalidomide and lenalidomide and proteasome inhibitors (PI) such as bortezomib are the few examples of these novel agents [45]. However, nearly all MM patients eventually relapse. High rates of relapse suggest that clinically undetectable minimal residual disease persists after treatment, and proliferation of the remaining myeloma cells ultimately results in relapse $[18,46]$. Therefore, sensitive and specific early diagnostic and prognostic biomarkers remain necessary for MM.

Molecular chaperone grp94 [47], also known as gp96 [48], endoplasmin [49], ERp99 [50], and HSP90b1 [51], is an endoplasmic reticulum (ER) paralog of HSP90. grp94 is a key downstream chaperone to mediate unfolded protein response (UPR) [52]. UPR is an evolutionally conserved mechanism that maintains protein quality control in the secretory pathway. Accumulation of misfolded proteins in the ER triggers the activation of three well-known pathways: activating transcription factor 6 (ATF6), the double-stranded RNA-activated protein kinase-like ER kinase (PERK), and the spliced form of Xbox binding protein 1 (XBP1s). These induce the expression of the major ER heat shock proteins including grp94, grp78, and calreticulin, which together enhance the protein folding machinery $[53,54]$. The pathogenesis of MM is closely linked to dysregulated UPR in the ER [55]. Constitutive activation of UPR in mice, as demonstrated by transgenic expression of a master UPR transcription factor XBP1s, causes myeloma [56]. Our recent study has shown that the persistence of plasma cells as well as the development of myeloma in XBP1s-transgenic mice is critically dependent on grp94 [57]. However, the role of grp94 in the initiation and progression of human MM is still unknown. In this study, we examined the expression levels of grp94 in BMPCs from patients with plasma cell disorders and non-plasma cell diseases. We found that high expression of grp94 in BMPCs is a novel molecular hallmark of MM.

\section{Results}

\section{Patient characteristics}

Patient characteristics in this study are summarized in Table 1. The median age was 57 years (range 32-75) for MM patients, 62 years (range 54-72) for MGUS/SMM 
Table 1 Clinical characteristics of patients

\begin{tabular}{llll}
\hline & MM & MGUS and SMM & NPC \\
& $(n)$ & $(n)$ & $(n)$ \\
\hline Total & 20 & 7 & 15 \\
Male & 12 & 2 & 12 \\
Female & 8 & 5 & 3 \\
Range of age (years) & $32-75$ & $54-72$ & $38-76$ \\
ISS & & & \\
Stage I & 4 & & \\
Stage II & 1 & & \\
Stage III & 13 & & \\
Type of monoclonal component & & & \\
IgG, K & 10 & 3 & \\
IgG, $\lambda$ & 1 & 2 & \\
IgA & 2 & 1 & \\
Light chain & 7 & 1 & \\
Non-secretory & 0 & & \\
B2 microglobulin & 5.8 & & \\
Median number of prior regimens & 2 & & \\
Auto HSCT & 3 & & \\
Prior bortezomib & 14 & & \\
Prior lenalidomide & 9 & & \\
Prior thalidomide & 2 & & \\
\hline
\end{tabular}

patients, and 63 years (range 38-76) for NPC patients. The male-to-female ratios were 1.5:1 in MM, 1:2.5 in MGUS/SMM, and 4:1 in NPC. Based on the ISS staging system [32], 4 MM patients were in stage I, 1 patient in stage II, and 13 patients in stage III at the time of diagnosis. The monoclonal component was of IgGk type in ten patients, IgG $\lambda$ type in one patient, IgA type in two patients, and light chain disease in seven patients. The median number of prior treatment regimens for $\mathrm{MM}$ was two. Three MM patients were treated with autologous hematopoietic cell transplantation. Fifteen NPC patients include 5 diffuse large B cell lymphoma (DLBCL) patients with active disease, 2 acute myelogenous leukemia (AML) patients with active disease, 3 AML patients with no disease, 1 mantle cell lymphoma (MCL) patient with active disease, 1 marginal zone $B$ cell lymphoma (MZL) patient with stage IV active disease, 1 Hodgkin lymphoma (HL) patient with active disease, 1 aplastic anemia (AA) patient, and 1 carcinoma patient with gastrointestinal (GI) primary.

\section{grp94 is preferentially expressed in plasma cells}

We first examined the protein expression level of grp94 on various cellular populations in the bone marrow (BM) using flow cytometry after intracellular staining with a highly specific monoclonal antibody against grp94.
Remarkably, although grp94 is thought to be constitutively expressed by all nucleated cells [52], we found that the expression level of grp94 in human BM cells in MM is extremely heterogeneous. Non-B cells (population I: CD38 ${ }^{-}$CD138 ${ }^{-}$cells), which are mostly myeloid cells, express grp94 at very low level. By comparison, plasma cells (population IV: CD $38^{\text {high }} \mathrm{CD} 138^{+}$cells) express the highest level of grp94, followed by pro- and pre-B cells (population III: CD38 ${ }^{\text {int }} \mathrm{CD} 138^{-}$cells) and naïve B cells (population II: CD38 ${ }^{\text {low }} \mathrm{CD} 138^{-}$cells) (Fig. 1a, b).

We next corroborated our findings by determining the mRNA expression level of grp94 in BMPCs in MM patients by real-time RT-PCR as well as Western blot. We isolated $\mathrm{CD} 138^{+} \mathrm{PCs}$ and $\mathrm{CD} 138^{-}$non-plasma cells from BM cells from MM patients using magnetic beads, followed by purification of total mRNA and real-time RT-PCR using primers specific for human grp94 cDNA. $18 \mathrm{~S}$ ribosomal RNA was used as an internal control. We found that grp94 transcript level in CD $138^{+}$BMPCs was more than 15-fold higher than $\mathrm{CD} 138^{-}$cells (Fig. 2a). Consistently, the significant high expression level of grp94 in CD $138^{+}$PCs was also confirmed by Western blot (Fig. 2b).

\section{grp94 is highly expressed in malignant plasma cells in MM}

We next determined if the high level of grp94 expression is unique to BMPCs of MM patients by broadening our analysis to patients with MGUS/SMM as well as NPC. As expected, BMPCs from MM patients expressed a significantly higher level of grp94, compared with BMPCs from patients with MGUS/SMM and NPC (Fig. 3a). CD138 (syndecan-1) is a specific marker of human plasma cells [58]. We found indeed that the expression of grp94 paralleled with CD138 level (Fig. 3c, left panel) in MM patients. However, while grp94 expression level separated out MGUS/SMM from NPCs, CD138 level failed to do so (Fig. 3a, b), demonstrating that grp94 expression is a better diagnostic marker than CD138 for malignant plasma cell disorder.

\section{Higher expression of grp94 is associated with worse disease in MM}

We next examined if the grp94 expression in malignant plasma cells in MM patients has any prognostic significance, by comparing grp94 expression level in BMPCs with the ISS staging status [32]. We found that the expression level of grp94 correlated with the clinical outcomes of MM patients. grp94 expression is significantly elevated in plasma cells from stage III MM patients compared to the ones at the stage I/II (Fig. 4). Collectively, we have demonstrated that high level of grp94 expression is not only a molecular hallmark unique to 
A
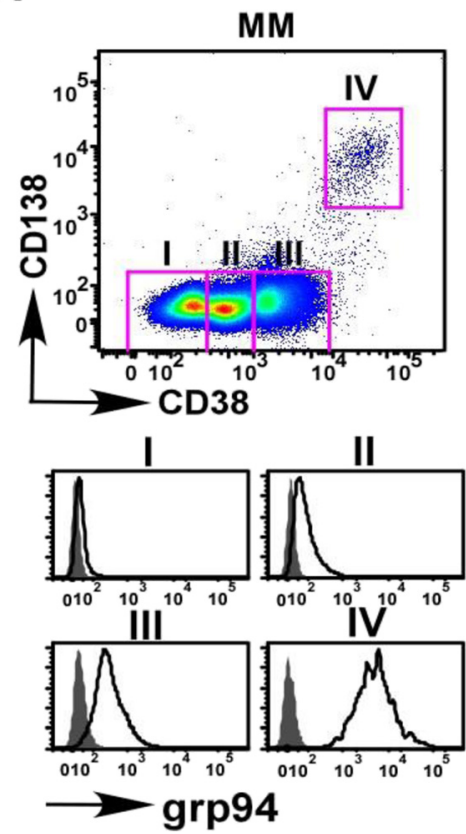

B

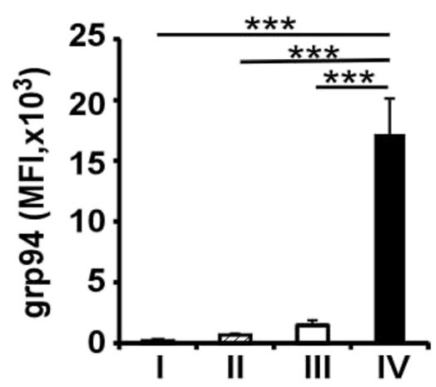

Fig. 1 grp94 is preferentially expressed on plasma cells. a Human bone marrow cells were analyzed and representative FACS plots of different populations in MM. Histograms show the intracellular staining of grp94 on different populations (open histogram indicates grp94; shaded histogram, isotype control). Population / CD38- CD138- cells (non-B cells), population // CD $38^{\text {low }} \mathrm{CD} 138^{-}$cells (naïve B cells), population III CD $38^{\text {int }} \mathrm{CD} 138^{-}$cells (pro- and pre-B cells), population IV CD38 ${ }^{\text {high }} \mathrm{CD} 138^{+}$cells (plasma cells). b Quantification of grp94 expression in different populations of bone marrow from the patients with MM. Error bars indicate standard error of the mean. ${ }^{* * *} p<0.001$

BMPCs of MM patients but it also predicts poorer clinical outcomes in MM patients.

\section{Discussion}

MM is characterized by the infiltration of bone marrow with clonal plasma cells that secrete a monoclonal protein in the majority of patients [59]. The hallmark of the pathology is the overproduction of a secreted protein by a malignant plasma cell population, and UPR plays critical roles in the pathogenesis of MM. grp94 is a key downstream chaperone in the ER and mediates UPR, but its roles in human MM was unexplored [52, 60]. In this study, we discovered for the first time that human plasma cells express significantly high level of grp94, when compared with other cellular populations, indicating that the plasma cells are under active ER stress.

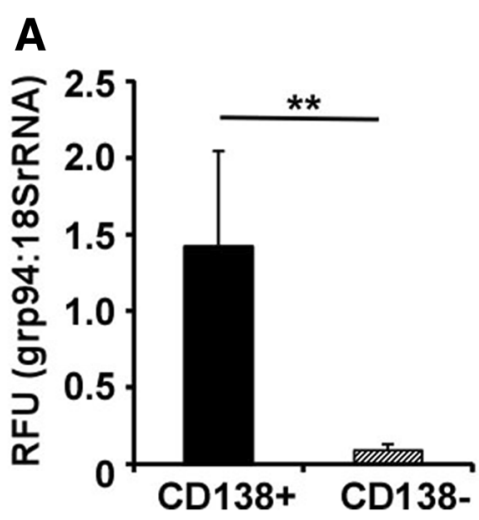

B

CD138+ CD138-
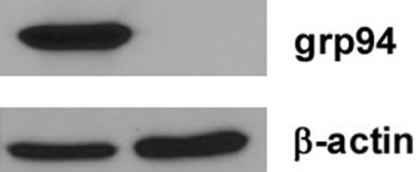

Fig. 2 grp94 is highly expressed in plasma cells. a Q-RT-PCR analysis of grp94 mRNA in CD138 ${ }^{+}$plasma cells and CD138 ${ }^{-}$non-plasma cells. 18S ribosomal RNA was used as an internal control. The quantity of transcripts for respective genes was expressed as a relative fluorescence unit (RFU), calculated based on the number of cycles to reach the threshold of detection (Ct) using the formula of $2^{\Delta \mathrm{Ct}}\left(\mathrm{Ct}{ }_{\text {interest }}-\mathrm{Ct}{ }_{18 \mathrm{~s}} \mathrm{RNA}\right)$. $\mathbf{b}$ Western blot for grp94 of CD138 ${ }^{+}$plasma cells and CD138 non-plasma cells. $\beta$-actin was blotted to indicate equal loading of cell lysates 
A

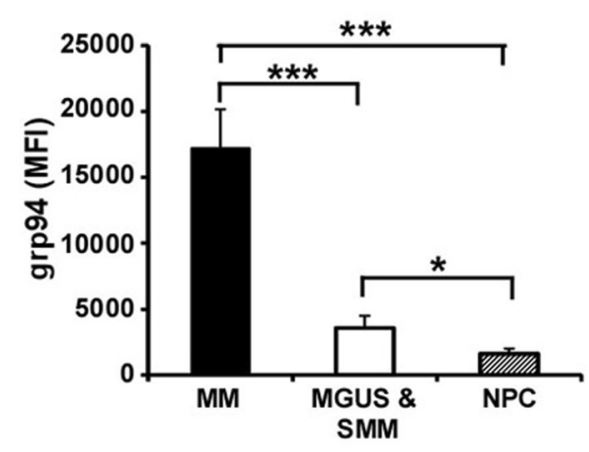

C

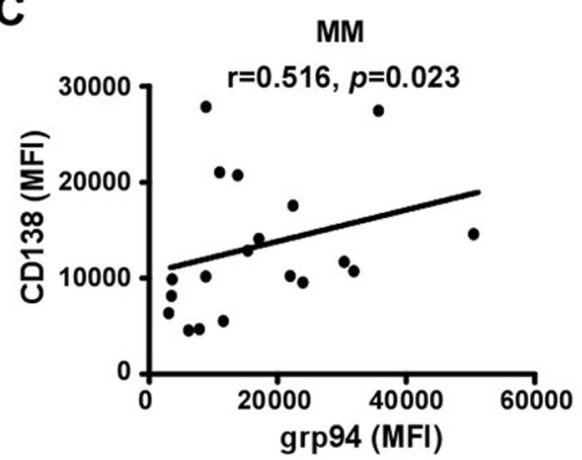

B
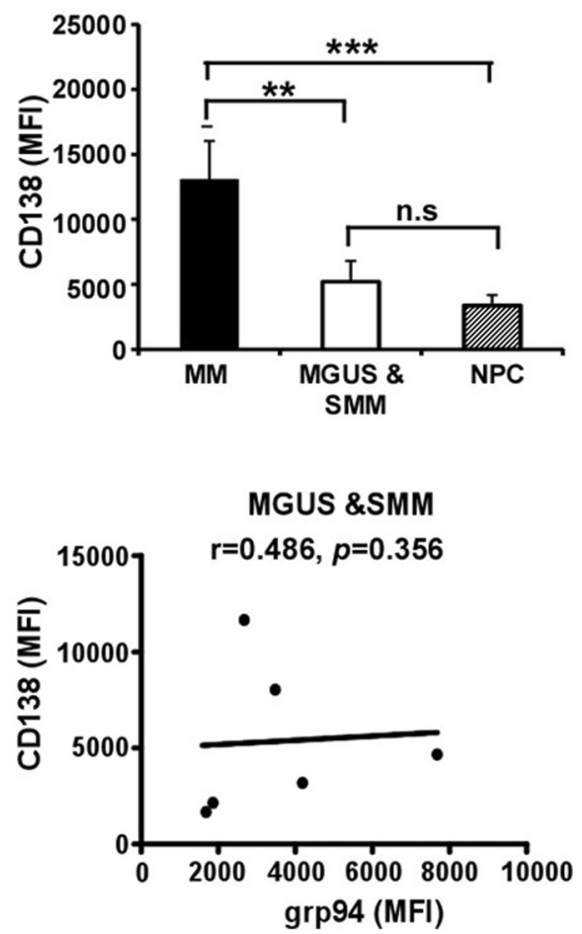

Fig. 3 grp94 is highly expressed on malignant plasma cells in MM. a Quantification of grp94 expression in the plasma cells from the patients with MM, MGUS/SMM, and NPC. Error bars indicate standard error of the mean. ${ }^{*} p<0.05,{ }^{* *} p<0.01,{ }^{* *} p<0.001$. b Quantification of CD138 expression in the plasma cells from the patients with MM, MGUS/SMM, and NPC. Error bars indicate standard error of the mean. ${ }^{* *} p<0.01,{ }^{* * *} p<$ 0.001. c Correlations between grp94 expression and CD138 expression in plasma cells from patients with MM (left panel) and patients with MGUS/SMM (right panel)

More importantly, we found that grp94 is highly expressed in malignant plasma cells in MM, when compared with MGUS/SMM and NPC. Our observation is consistent with the notion that grp94 is critical for malignant plasma cell survival and persistence [57], but not for benign plasma cells [61]. CD138 is commonly expressed by plasma cells, but it is not a unique diagnostic marker for MM [58]. A recent study showed that the serum level of CD138 was significantly higher in active MM than that in MGUS [62]. Consistent to this study, we found that CD138 is highly expressed on plasma cells from the MM patients, when compared with patients with MGUS/SMM and NPC. We also observed that grp94 expression significantly correlated with the CD138 expression in plasma cells from patients with MM, but not with MGUS/SMM. Further studies with a larger cohort of patients shall solidify this conclusion. Interestingly, despite a small sample size, we discovered that the grp94 expression in malignant plasma cells likely has prognostic significance. Its level is significantly elevated in plasma cells from the ISS stage III MM patients in comparison with the stage I/II. A future perspective study is warranted to further establish both the

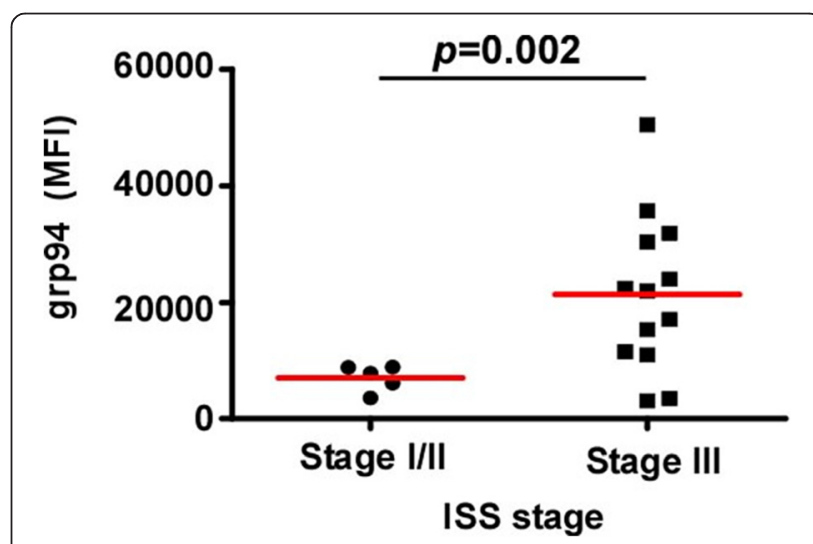

Fig. 4 Higher expression of grp94 is associated with worse disease in MM. Compare grp94 expression level in malignant plasma cells from MM patients with different ISS stages. The grp94 expression level is significantly higher in malignant plasma cells from stage III MM patients than that from stage I/II MM patients. $(p=0.002)$ 
diagnostic and prognostic value of BMPC-intrinsic grp94 expression in $\mathrm{MM}$ and other plasma cell disorders.

Among many client proteins chaperoned by grp94 [52, 61, 63, 64], Wnt co-receptor low-density lipoprotein receptor-related protein 6 (LRP6) for canonical Wnt signaling depends exclusively on grp94 for folding $[57,65]$. Our study thus may add significantly to the emerging roles of Wnt signaling in MM [66-68]. Human MM cells appear to have evidence of active Wnt signaling by overexpressing $\beta$-catenin which promotes proliferation of malignant cells [69]. Blocking $\beta$-catenin with smallmolecule inhibitors, AV-65 [68] or PKF115-584 [66, 67], specifically inhibits MM cell proliferation. Our previous study showed that in the absence of grp94, MM cells undergo mitotic catastrophe and apoptosis which correlated with decreased expression of survivin, a downstream target molecule of Wnt signaling [57].

Finally, grp94 might promote the pathogenesis of MM through folding other client proteins such as integrins, IGF, and Toll-like receptors [61, 63, 64]. Intriguingly, grp94-selective inhibitors are already in early development $[70,71]$. Our study may therefore pave a way for developing grp94-targeted strategy for the treatment of MM.

\section{Conclusions}

In summary, we found that grp94 is highly expressed in malignant plasma cells in multiple myeloma. The higher level of grp94 is significantly associated with worse clinical stage in this disease. Our data demonstrated that grp94 is not only a specific diagnostic and prognostic biomarker but also a therapeutic target in multiple myeloma.

\section{Methods}

\section{Study subjects}

Forty-two patients at the Hollings Cancer Center, Medical University of South Carolina, were enrolled in this study. Bone marrow samples were taken from 20 patients with MM, 7 patients diagnosed with MGUS or SMM, and 15 patients with NPC, which include DLBCL, AML, MCL, MZL, HL, AA, and GI primary carcinoma. This study was conducted in accordance with the ethical guidelines and was approved by the Institutional Review Board of the Medical University of South Carolina. Informed consent was obtained from all patients.

\section{Reagents}

Antibodies used for flow cytometry were obtained from BD Biosciences (Mountain View, CA) and eBioscience (San Diego, CA). Antibodies against grp94 Ab (9G10) was bought from Enzo Life Sciences, Inc. (Farmingdale, $\mathrm{NY}$ ), and $\beta$-actin $\mathrm{Ab}$ (AC-74) was purchased from
Sigma-Aldrich (St Louis, MO). All other chemicals were obtained from Sigma-Aldrich (St Louis, MO) and Fisher Scientific (Pittsburgh, PA).

\section{Isolation of bone marrow cells}

Add Histopaque-1077 to a $15-\mathrm{mL}$ conical centrifuge tube and warm to room temperature. Carefully add anticoagulated bone marrow aspirate onto the Histopaque1077 at 1:1 ratio (Sigma-Aldrich, St Louis, MO). Centrifuge at $400 \mathrm{~g}$ for $30 \mathrm{~min}$ at room temperature. After centrifugation, aspirate the upper layer with a Pasteur pipette to within $0.5 \mathrm{~cm}$ of the opaque interface containing bone marrow cells. Discard upper layer and transfer the opaque interface into a clean conical centrifuge tube. Wash the cells by adding $10 \mathrm{~mL}$ PBS and centrifuge at $1500 \mathrm{rpm}$ for $5 \mathrm{~min}$.

\section{Flow cytometry}

Surface staining of cells and flow cytometry were done as described [64, 72, 73]. To stain grp94 intracellularly, cells were fixed in $4 \%$ paraformaldehyde and permeablized in ice-cold methanol. Cells were acquired on FACSVerse (Becton Dickinson, Franklin Lakes, NJ), and results were analyzed with FloJo software (Tree Star, Ashland, OR).

\section{Quantitative RT-PCR}

$\mathrm{CD}_{138^{+}}$and $\mathrm{CD} 138^{-}$cells were isolated from BM by using human CD138 positive selection kit (StemCell Technologies Inc., Vancouver, Canada). Total RNA was extracted with the RNeasy Mini kit according to the manufacturer's protocol (Qiagen, Valencia, CA). First-strand cDNA was synthesized with Superscript II (Invitrogen, Carlsbad, CA). cDNA was quantified by Q-PCR with Bio-Rad CFX Connect Real-Time System. Q-PCR data were analyzed with the corresponding software, and the number of PCR cycles to reach the threshold of detection (CT) was calculated. Samples were run in duplicates. $18 \mathrm{~S}$ ribosomal RNA (18S rRNA) was used as an internal control. The primer sequences are as follows. Human grp94F, GCTTCG GTCAGGGTATCTTT; human grp94R, AGGCTCTTC TTCCACCTTTG; $18 \mathrm{~S}$ rRNA F, CGGCTACCACATC CAAGGAA; 18S rRNA R, GCTGGAATTACCGCGGCT.

\section{Protein extraction and Western blot}

Protein extraction and immunoblot were performed as described previously [74]. Briefly, cells were washed three times with ice-cold PBS and lysed in radioimmunoprecipitation assay (RIPA) lysis buffer ( $0.01 \mathrm{M}$ sodium phosphate, pH 7.2, $150 \mathrm{mM} \mathrm{NaCl}, 2$ mM EDTA, 1 \% NP-40, 1 \% sodium deoxycholate, $0.1 \%$ SDS, $2 \mathrm{mM}$ AEBSF, $130 \mathrm{mM}$

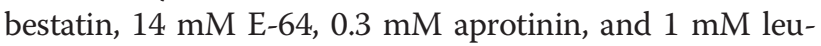
peptin). Total cell lysates was resolved on denaturing and reducing $10 \%$ SDS-PAGE, and the proteins were 
transferred from the gel onto Immobilon-P membranes. The membrane was blocked with $5 \%$ nonfat milk in PBS and then incubated with different Abs, followed by incubation with HRP-conjugated secondary Ab. Protein bands were visualized by using enhanced chemiluminescent substrate (Pierce, Rockford, IL).

\section{Statistical analysis}

Error bars represent the standard error of the mean (SEM). Independent-samples $t$ tests or ANOVA were used to compare variables between different groups. Correlations between variables were assessed using Pearson correlation analysis. All statistical analyses were performed using Prism 5 software. Values of $P$ less than 0.05 were considered to represent statistically significant differences.

\begin{abstract}
Abbreviations
MM: multiple myeloma; MGUS: monoclonal gammopathy of undetermined significance; SMM: smoldering multiple myeloma; NPC: non-plasma cell disorder; ER: endoplasmic reticulum; HSP: heat shock protein; UPR: unfolded protein response; ATF6: activating transcription factor 6; PERK: the doublestranded RNA-activated protein kinase-like ER kinase; XBP1s: spliced form of X-box binding protein 1; BM: bone marrow.
\end{abstract}

\section{Competing interests}

The authors declare that they have no competing interests.

\section{Authors' contributions}

BL conceived the idea, designed the study, and performed the experiments. SC, CW, FH, SJ, and BL analyzed and interpreted the data. SC and SJ obtained patient consent and primary human BM samples. SC and BL wrote the manuscript. All authors read and approved the final manuscript.

\section{Acknowledgements}

This study was supported in part by the South Carolina Clinical \& Translational Research Institute at the Medical University of South Carolina KL2RR029880 and UL1RR029882 (to BL), the American Cancer Society Institutional Research Grant from the American Cancer Society IRG-97-219-14 (to BL), and the Medical University of South Caroline Bridge Funding and Start Up Fund (to BL). This study was also supported in part by the Biorepository \& Tissue Analysis and Flow Cytometry \& Cell Sorting Unit Shared Resource, Hollings Cancer Center, Medical University of South Carolina (P30 CA138313).

\section{Author details}

'Hollings Cancer Center, 86 Jonathan Lucas Street, Charleston, SC 29425, USA. ${ }^{2}$ Department of Microbiology and Immunology, Medical University of South Carolina, 86 Jonathan Lucas Street, Charleston, SC 29425, USA. ${ }^{3}$ Division of Hematology and Oncology, Department of Medicine, Medical University of South Carolina, Charleston, SC 29425, USA.

Received: 23 May 2015 Accepted: 17 June 2015

Published online: 25 June 2015

\section{References}

1. Munshi NC, Anderson KC. New strategies in the treatment of multiple myeloma. Clin Cancer Res. 2013;19(13):3337-44.

2. Garcia MK, Cohen L, Guo Y, Zhou Y, You B, Chiang J, et al. Electroacupuncture for thalidomide/bortezomib-induced peripheral neuropathy in multiple myeloma: a feasibility study. J Hematol Oncol. 2014;7:41.

3. Han $\mathrm{K}, \mathrm{Xu}$ X, Chen G, Zeng Y, Zhu J, Du X, et al. Identification of a promising $\mathrm{PI} 3 \mathrm{~K}$ inhibitor for the treatment of multiple myeloma through the structural optimization. J Hematol Oncol. 2014;7:9.

4. Hou J, Du X, Jin J, Cai Z, Chen F, Zhou DB, et al. A multicenter, open-label, phase 2 study of lenalidomide plus low-dose dexamethasone in Chinese patients with relapsed/refractory multiple myeloma: the MM-021 trial. J Hematol Oncol. 2013;6:41.

5. Kharfan-Dabaja MA, Hamadani M, Reljic T, Nishihori T, Bensinger W, Djulbegovic B, et al. Comparative efficacy of tandem autologous versus autologous followed by allogeneic hematopoietic cell transplantation in patients with newly diagnosed multiple myeloma: a systematic review and meta-analysis of randomized controlled trials. J Hematol Oncol. 2013;6:2.

6. Phillip CJ, Zaman S, Shentu S, Balakrishnan K, Zhang J, Baladandayuthapani $V$, et al. Targeting MET kinase with the small-molecule inhibitor amuvatinib induces cytotoxicity in primary myeloma cells and cell lines. J Hematol Oncol. 2013;6:92.

7. Schmitt T, Goldschmidt H, Neben K, Freiberger A, Husing J, Gronkowski M, et al. Aprepitant, granisetron, and dexamethasone for prevention of chemotherapy-induced nausea and vomiting after high-dose melphalan in autologous transplantation for multiple myeloma: results of a randomized, placebo-controlled phase III trial. J Clin Oncol. 2014;32(30):3413-20.

8. Papadopoulos KP, Siegel DS, Vesole DH, Lee P, Rosen ST, Zojwalla N, et al. Phase I study of 30-minute infusion of carfilzomib as single agent or in combination with low-dose dexamethasone in patients with relapsed and/ or refractory multiple myeloma. J Clin Oncol. 2015;33(7):732-9.

9. Roussel M, Lauwers-Cances V, Robillard N, Hulin C, Leleu X, Benboubker L, et al. Front-line transplantation program with lenalidomide, bortezomib, and dexamethasone combination as induction and consolidation followed by lenalidomide maintenance in patients with multiple myeloma: a phase II study by the Intergroupe Francophone du Myelome. J Clin Oncol. 2014;32(25):2712-7.

10. Palumbo A, Bringhen S, Larocca A, Rossi D, Di Raimondo F, Magarotto V, et al. Bortezomib-melphalan-prednisone-thalidomide followed by maintenance with bortezomib-thalidomide compared with bortezomibmelphalan-prednisone for initial treatment of multiple myeloma: updated follow-up and improved survival. J Clin Oncol. 2014;32(7):634-40.

11. Lonial S, Dimopoulos M, Palumbo A, White D, Grosicki S, Spicka I, et al. Elotuzumab therapy for relapsed or refractory multiple myeloma. N Engl J Med. 2015.

12. Mateos MV, San Miguel JF. How should we treat newly diagnosed multiple myeloma patients? Hematology Am Soc Hematol Educ Program. 2013;2013:488-95.

13. Rajkumar SV. Treatment of multiple myeloma. Nat Rev Clin Oncol. 2011;8(8):479-91.

14. Rajkumar SV, Gahrton G, Bergsagel PL. Approach to the treatment of multiple myeloma: a clash of philosophies. Blood. 2011;118(12):3205-11.

15. Vincent RS. Multiple myeloma: 2014 Update on diagnosis, risk-stratification, and management. Am J Hematol. 2014:89(10):999-1009.

16. Siegel R, Ma J, Zou Z, Jemal A. Cancer statistics, 2014. CA Cancer J Clin 2014;64(1):9-29.

17. Palumbo A, Anderson K. Multiple myeloma. N Engl J Med. 2011;364(11):1046-60.

18. Kellner J, Liu B, Kang Y, Li Z. Fact or fiction-identifying the elusive multiple myeloma stem cell. J Hematol Oncol. 2013;6:91.

19. Rajkumar SV, Dimopoulos MA, Palumbo A, Blade J, Merlini G, Mateos MV et al. International Myeloma Working Group updated criteria for the diagnosis of multiple myeloma. Lancet Oncol. 2014;15(12):e538-48.

20. Landgren O, Kyle RA, Pfeiffer RM, Katzmann JA, Caporaso NE, Hayes RB, et al. Monoclonal gammopathy of undetermined significance (MGUS) consistently precedes multiple myeloma: a prospective study. Blood. 2009:113(22):5412-7.

21. Weiss BM, Abadie J, Verma P, Howard RS, Kuehl WM. A monoclonal gammopathy precedes multiple myeloma in most patients. Blood. 2009;113(22):5418-22.

22. Kyle RA, Remstein ED, Therneau TM, Dispenzieri A, Kurtin PJ, Hodnefield JM, et al. Clinical course and prognosis of smoldering (asymptomatic) multiple myeloma. N Engl J Med. 2007;356(25):2582-90.

23. Rajkumar SV. Preventive strategies in monoclonal gammopathy of undetermined significance and smoldering multiple myeloma. Am J Hematol. 2012;87(5):453-4.

24. Rajkumar SV, Merlini G, San Miguel JF. Haematological cancer: redefining myeloma. Nat Rev Clin Oncol. 2012;9(9):494-6.

25. Landgren O, Waxman AJ. Multiple myeloma precursor disease. JAMA. 2010;304(21):2397-404

26. Durie BG, Salmon SE. A clinical staging system for multiple myeloma. Correlation of measured myeloma cell mass with presenting clinical features, response to treatment, and survival. Cancer. 1975;36(3):842-54. 
27. Bataille R, Durie BG, Grenier J. Serum beta2 microglobulin and survival duration in multiple myeloma: a simple reliable marker for staging. $\mathrm{Br} J$ Haematol. 1983;55(3):439-47.

28. Durie BG, Stock-Novack D, Salmon SE, Finley P, Beckord J, Crowley J, et al. Prognostic value of pretreatment serum beta 2 microglobulin in myeloma: a Southwest Oncology Group Study. Blood. 1990;75(4):823-30.

29. Bataille R, Durie BG, Grenier J, Sany J. Prognostic factors and staging in multiple myeloma: a reappraisal. J Clin Oncol. 1986;4(1):80-7.

30. Greipp PR, Lust JA, O'Fallon WM, Katzmann JA, Witzig TE, Kyle RA. Plasma cell labeling index and beta 2-microglobulin predict survival independent of thymidine kinase and C-reactive protein in multiple myeloma. Blood. 1993;81(12):3382-7.

31. San Miguel JF, Garcia-Sanz R, Gonzalez M, Moro MJ, Hernandez JM, Ortega $F$, et al. A new staging system for multiple myeloma based on the number of S-phase plasma cells. Blood. 1995;85(2):448-55.

32. Greipp PR, San Miguel J, Durie BG, Crowley JJ, Barlogie B, Blade J, et al. International staging system for multiple myeloma. J Clin Oncol. 2005;23(15):3412-20.

33. Boyd KD, Ross FM, Chiecchio L, Dagrada GP, Konn ZJ, Tapper WJ, et al. A novel prognostic model in myeloma based on co-segregating adverse FISH lesions and the ISS: analysis of patients treated in the MRC Myeloma IX trial. Leukemia. 2012:26(2):349-55.

34. Avet-Loiseau H, Durie BG, Cavo M, Attal M, Gutierrez N, Haessler J, et al. Combining fluorescent in situ hybridization data with ISS staging improves risk assessment in myeloma: an International Myeloma Working Group collaborative project. Leukemia. 2013;27(3):711-7.

35. Fonseca R, Bergsagel PL, Drach J, Shaughnessy J, Gutierrez N, Stewart AK, et al. International Myeloma Working Group molecular classification of multiple myeloma: spotlight review. Leukemia. 2009;23(12):2210-21.

36. Ludwig H, Milosavljevic D, Zojer N, Faint JM, Bradwell AR, Hubl W, et al. Immunoglobulin heavy/light chain ratios improve paraprotein detection and monitoring, identify residual disease and correlate with survival in multiple myeloma patients. Leukemia. 2013;27(1):213-9.

37. Micallef J, Dharsee M, Chen J, Ackloo S, Evans K, Qiu L, et al. Applying mass spectrometry based proteomic technology to advance the understanding of multiple myeloma. J Hematol Oncol. 2010;3:13.

38. Zheng W, Liu D, Fan X, Powers L, Goswami M, Hu Y, et al. Potential therapeutic biomarkers in plasma cell myeloma: a flow cytometry study. Cytometry B Clin Cytom. 2013;84(4):222-8.

39. Vandyke K, Chow AW, Williams SA, To LB, Zannettino AC. Circulating Ncadherin levels are a negative prognostic indicator in patients with multiple myeloma. Br J Haematol. 2013;161(4):499-507.

40. Qu X, Zhao M, Wu S, Yu W, Xu J, Xu J, et al. Circulating microRNA 483-5p as a novel biomarker for diagnosis survival prediction in multiple myeloma. Med Oncol. 2014;31(10):219.

41. Bolomsky A, Hose D, Schreder M, Seckinger A, Lipp S, Klein B, et al. Insulin like growth factor binding protein 7 (IGFBP7) expression is linked to poor prognosis but may protect from bone disease in multiple myeloma. J Hematol Oncol. 2015;8(1):10.

42. Kumar SK, Rajkumar SV, Dispenzieri A, Lacy MQ, Hayman SR, Buadi FK, et al. Improved survival in multiple myeloma and the impact of novel therapies. Blood. 2008;111(5):2516-20.

43. Pulte $\mathrm{D}$, Gondos A, Brenner $\mathrm{H}$. Improvement in survival of older adults with multiple myeloma: results of an updated period analysis of SEER data. Oncologist. 2011;16(11):1600-3.

44. Attal M, Harousseau JL, Stoppa AM, Sotto JJ, Fuzibet JG, Rossi JF, et al. A prospective, randomized trial of autologous bone marrow transplantation and chemotherapy in multiple myeloma. Intergroupe Francais du Myelome. N Engl J Med. 1996;335(2):91-7.

45. Lonial S, Boise LH. The future of drug development and therapy in myeloma. Semin Oncol. 2013;40(5):652-8.

46. Noonan K, Borrello I. The immune microenvironment of myeloma. Cancer Microenviron. 2011;4(3):313-23.

47. Lee AS, Delegeane A, Scharff D. Highly conserved glucose-regulated protein in hamster and chicken cells: preliminary characterization of its CDNA clone. Proc Natl Acad Sci U S A. 1981;78(8):4922-5.

48. Srivastava PK, DeLeo AB, Old LJ. Tumor rejection antigens of chemically induced sarcomas of inbred mice. Proc Natl Acad Sci U S A 1986:83(10):3407-11.
49. Koch G, Smith M, Macer D, Webster P, Mortara R. Endoplasmic reticulum contains a common, abundant calcium-binding glycoprotein, endoplasmin. J Cell Sci. 1986;86:217-32.

50. Lewis MJ, Mazzarella RA, Green M. Structure and assembly of the endoplasmic reticulum. The synthesis of three major endoplasmic reticulum proteins during lipopolysaccharide-induced differentiation of murine lymphocytes. J Biol Chem. 1985;260(5):3050-7.

51. Chen B, Piel WH, Gui L, Bruford E, Monteiro A. The HSP90 family of genes in the human genome: insights into their divergence and evolution. Genomics. 2005;86(6):627-37.

52. Yang Y, Li Z. Roles of heat shock protein gp96 in the ER quality control: redundant or unique function? Mol Cells. 2005;20(2):173-82.

53. Malhotra JD, Kaufman RJ. The endoplasmic reticulum and the unfolded protein response. Semin Cell Dev Biol. 2007;18(6):716-31.

54. Li X, Zhang K, Li Z. Unfolded protein response in cancer: the physician's perspective. J Hematol Oncol. 2011:4:8.

55. Bagratuni T, Wu P, Gonzalez de Castro D, Davenport EL, Dickens NJ, Walker BA, et al. XBP1s levels are implicated in the biology and outcome of myeloma mediating different clinical outcomes to thalidomide-based treatments. Blood. 2010;116(2):250-3.

56. Carrasco DR, Sukhdeo K, Protopopova M, Sinha R, Enos M, Carrasco DE, et al. The differentiation and stress response factor XBP-1 drives multiple myeloma pathogenesis. Cancer Cell. 2007:11(4):349-60.

57. Hua Y, White-Gilbertson S, Kellner J, Rachidi S, Usmani SZ, Chiosis G, et al. Molecular chaperone gp96 is a novel therapeutic target of multiple myeloma. Clin Cancer Res. 2013;19(22):6242-51.

58. O'Connell FP, Pinkus JL, Pinkus GS. CD138 (syndecan-1), a plasma cell marker immunohistochemical profile in hematopoietic and nonhematopoietic neoplasms. Am J Clin Pathol. 2004;121(2):254-63.

59. Chng WJ, Dispenzieri A, Chim CS, Fonseca R, Goldschmidt H, Lentzsch S, et al. IMWG consensus on risk stratification in multiple myeloma. Leukemia. 2014;28(2):269-77.

60. White-Gilbertson S, Hua Y, Liu B. The role of endoplasmic reticulum stress in maintaining and targeting multiple myeloma: a double-edged sword of adaptation and apoptosis. Front Genet. 2013;4:109.

61. Liu B, Li Z. Endoplasmic reticulum HSP90b1 (gp96, grp94) optimizes B-cell function via chaperoning integrin and TLR but not immunoglobulin. Blood. 2008;112(4):1223-30.

62. Minarik J, Pika T, Bacovsky J, Petrova P, Langova K, Scudla V. Prognostic value of hepatocyte growth factor, syndecan-1, and osteopontin in multiple myeloma and monoclonal gammopathy of undetermined significance. Sci World J. 2012;2012:356128.

63. Staron M, Yang Y, Liu B, Li J, Shen Y, Zuniga-Pflucker JC, et al. gp96, an endoplasmic reticulum master chaperone for integrins and Toll-like receptors, selectively regulates early $\mathrm{T}$ and $\mathrm{B}$ lymphopoiesis. Blood. 2010;115(12):2380-90.

64. Yang Y, Liu B, Dai J, Srivastava PK, Zammit DJ, Lefrancois L, et al. Heat shock protein gp96 is a master chaperone for toll-like receptors and is important in the innate function of macrophages. Immunity. 2007;26(2):215-26.

65. Liu B, Staron M, Hong F, Wu BX, Sun S, Morales C, et al. Essential roles of grp94 in gut homeostasis via chaperoning canonical Wnt pathway. Proc Natl Acad Sci U S A. 2013;110(17):6877-82.

66. Sukhdeo K, Mani M, Zhang Y, Dutta J, Yasui H, Rooney MD, et al. Targeting the beta-catenin/TCF transcriptional complex in the treatment of multiple myeloma. Proc Natl Acad Sci U S A. 2007;104(18):7516-21.

67. Ashihara E, Kawata E, Nakagawa Y, Shimazaski C, Kuroda J, Taniguchi K, et al. beta-catenin small interfering RNA successfully suppressed progression of multiple myeloma in a mouse model. Clin Cancer Res. 2009;15(8):2731-8.

68. Yao H, Ashihara E, Strovel JW, Nakagawa Y, Kuroda J, Nagao R, et al. AV-65, a novel Wnt/beta-catenin signal inhibitor, successfully suppresses progression of multiple myeloma in a mouse model. Blood Cancer J. 2011;1(11), e43.

69. Derksen PW, Tjin E, Meijer HP, Klok MD, MacGillavry HD, van Oers MH, et al. Illegitimate WNT signaling promotes proliferation of multiple myeloma cells. Proc Natl Acad Sci U S A. 2004;101(16):6122-7.

70. Patel PD, Yan P, Seidler PM, Patel HJ, Sun W, Yang C, et al. Paralog-selective Hsp90 inhibitors define tumor-specific regulation of HER2. Nat Chem Biol. 2013;9(11):677-84.

71. Patel HJ, Patel PD, Ochiana SO, Yan P, Sun W, Patel MR, et al. Structureactivity relationship in a purine-scaffold compound series with selectivity for the endoplasmic reticulum hsp90 paralog grp94. J Med Chem. 2015;58(9):3922-43. 
72. Liu B, Staron M, Li Z. Murine but not human basophil undergoes cellspecific proteolysis of a major endoplasmic reticulum chaperone. PLOS ONE. 2012; $7(6): e 39442$.

73. Wu S, Hong F, Gewirth D, Guo B, Liu B, Li Z. The molecular chaperone gp96/GRP94 interacts with Toll-like receptors and integrins via its C-terminal hydrophobic domain. J Biol Chem. 2012;287(9):6735-42.

74. Liu B, Yang Y, Qiu Z, Staron M, Hong F, Li Y, et al. Folding of Toll-like receptors by the HSP90 paralogue gp96 requires a substrate-specific cochaperone. Nat Commun. 2010;1:79.

Submit your next manuscript to BioMed Central and take full advantage of:

- Convenient online submission

- Thorough peer review

- No space constraints or color figure charges

- Immediate publication on acceptance

- Inclusion in PubMed, CAS, Scopus and Google Scholar

- Research which is freely available for redistribution 